\title{
Title: Altered synaptic ingestion by human microglia in Alzheimer's disease
}

\section{Authors:}

Makis Tzioras ${ }^{1,2}$, Michael J.D. Daniels ${ }^{1,2}$, Declan King ${ }^{1,2}$, Karla Popovic ${ }^{1,2}$, Rebecca K. Holloway $^{3}$, Anna J. Stevenson ${ }^{1,2}$, Jane Tulloch ${ }^{1,2}$, Jothy Kandasamy ${ }^{4}$, Drahoslav Sokol ${ }^{4}$, Clare Latta $^{1,2}$, Jamie Rose ${ }^{1,2}$, Colin Smith ${ }^{5}$, Veronique E. Miron ${ }^{3}$, Christopher Henstridge ${ }^{6}$, Barry W. McColl $^{1,2 *}$, Tara L. Spires-Jones ${ }^{1,2 *}$

\section{Affiliations:}

${ }^{1}$ UK Dementia Research Institute, The University of Edinburgh, UK

${ }^{2}$ Centre for Brain Discovery Sciences, The University of Edinburgh, UK

${ }^{3}$ MRC Centre for Reproductive Health, The University of Edinburgh, UK

${ }^{4}$ Department of Clinical Neurosciences, Western General Hospital, Edinburgh, UK

${ }^{5}$ Centre for Clinical Brain Sciences, The University of Edinburgh, UK

${ }^{6}$ Division of Systems Medicine, School of Medicine, University of Dundee, Dundee, UK

*Correspondence to: Barry.McColl@,ed.ac.uk and Tara.Spires-Jones@ed.ac.uk

\section{Abstract:}

Synapse loss correlates strongly with cognitive decline in Alzheimer's disease, but the mechanisms underpinning this phenomenon remain unclear. Recent evidence from mouse models points to microglial cells as mediators of synapse removal, and human genetic evidence implicates microglia in disease risk. Here we demonstrate that microglia from human postmortem brain contain synaptic proteins and that greater amounts are observed in microglia from Alzheimer's compared to non-diseased brain tissue. Further, we observe that primary human adult microglia phagocytose synapses isolated from human brain, and that AD brain-derived synapses are phagocytosed more rapidly and abundantly than controls. Together, these data show that synapses in the human AD brain are more prone to ingestion by microglia. Our findings provide evidence from human tissue implicating altered microglial-mediated synaptic uptake in $30 \quad$ AD pathobiology.

One Sentence Summary: AD alters synapse ingestion by microglia 


\section{Main text:}

Alzheimer's disease (AD) is a lethal neurodegenerative disease affecting millions worldwide and is characterised by pathological protein aggregates and progressive cognitive decline. These protein aggregates, amyloid-beta $(\mathrm{A} \beta)$ plaques and tau tangles, are associated with synapse loss (1), which is the best-known correlate of cognitive decline in $\operatorname{AD}(2,3)$. Synapse pathology is proposed to contribute to disease pathogenesis, but the mechanisms underlying synapse degeneration remain unknown. Microglia, central nervous system-resident macrophages, have also been implicated as modulators of AD pathogenesis. This evidence stems from histopathological findings showing an accumulation of reactive microglia (4-6), as well as Genome-Wide Association Studies that identified multiple AD risk-factor genes expressed in microglia (7-9). During mouse brain development, microglia prune weak synapses and play a role in activitydependent sculpting of neural circuits (10-13). In mouse models of AD-like pathology, microglia have been proposed to contribute to synapse loss by aberrantly phagocytosing both healthy and dysfunctional synapses (14-17). While the evidence from these studies implicates microglia in synaptic loss, there is a key lack of evidence in humans and drug targets developed based on mouse work have thus far failed to translate to the clinic. Here, we provide evidence that microglia contain synaptic protein in aged human brain and that this is increased in AD brains compared to agematched controls, particularly surrounding A $\beta$ plaques. Further, we observe that cultured primary adult human microglia ingest synaptoneurosomes isolated from human brain with those from AD brain engulfed more readily than those derived from healthy brains. This provides key evidence from humans implicating altered synaptic phagocytosis by microglia in AD.

Given that microglia have been implicated as drivers of synapse loss in mouse models of AD-like pathology (18), we questioned if microglia ingest synapses in the human brain, and if so, is this process altered in AD. We examined human post-mortem tissue from individuals with late-stage $\mathrm{AD}$ and age-matched non-demented controls (NDCs) in two brain areas, the inferior temporal lobe (temporal cortex, BA20/21) which contains substantial A $\beta$ and tau pathology, and the primary visual cortex (occipital cortex, BA17) which is affected later in dementia and contains less pathology than temporal cortex even at end stages of disease (19) (information for human cases found at Table S1). We used immunofluorescence and confocal microscopy to examine whether 
the pre-synaptic maker synapsin I (SynI) was found colocalised with the microglial lysosomal marker CD68. Although CD68 labels both microglia and macrophages, in the cortical neuropil regions examined from our cases, we observe that CD68 positive cells were also positive for IBA1 and the microglial marker TMEM119 (20) (Figs. S1A\&B). We observed SynI signal localized within CD68+ microglia from both control and AD brains, suggesting synaptic internalisation occurs in the ageing brain independently of disease (Fig. 1A-C, D-F, G-I, J-L). However, there was significantly increased colocalisation of CD68 and SynI in AD compared to control brains in both brain regions examined (Fig. 1M, linear mixed-effects model on Tukey transformed data: effect of disease $\beta=0.03638$, stderr $=0.01210, p=0.00482$, pairwise post-hoc comparison of the model, $\mathrm{p}=0.0048$ ). There were no significant effects of brain region, APOE genotype, or sex in the colocalisation of SynI with CD68, despite extensive literature showing APOE4 carriers exhibit exacerbated synapse loss, neuroinflammation, and increased risk of late-onset AD, and females having a greater risk of developing $\operatorname{AD}(21,22)$. Since microgliosis is a prominent feature of the $\operatorname{AD}$ brain $(6,23)$, we quantified CD68 burdens (\% area). As expected, we found significantly higher CD68 burdens in AD in both the occipital and temporal lobes (Fig. 1N, linear mixed-effects model on Tukey transformed data: effect of disease $\beta=0.31725$, stderr $=0.08974, p=0.00116$; pairwise post-hoc comparison of the model, $\mathrm{p}=0.0012$ ). No differences were found between the two brain areas. It is unclear whether individual microglia internalise synapses during AD more readily, or whether the increased microglial burden results in greater overall synapse ingestion. To examine this, we normalised the area of colocalisation between CD68 and SynI by the area occupied by CD68. When normalised, we found no significant difference between AD and controls $(p=0.470)$ (Fig. 10). These data imply that the increased ingestion of synaptic protein by microglia in $\mathrm{AD}$ is due to increased numbers of microglia although we cannot exclude that hypertropy of microglia may account for the greater CD68 burden and thus reflect more synaptic protein per microglial cell. 

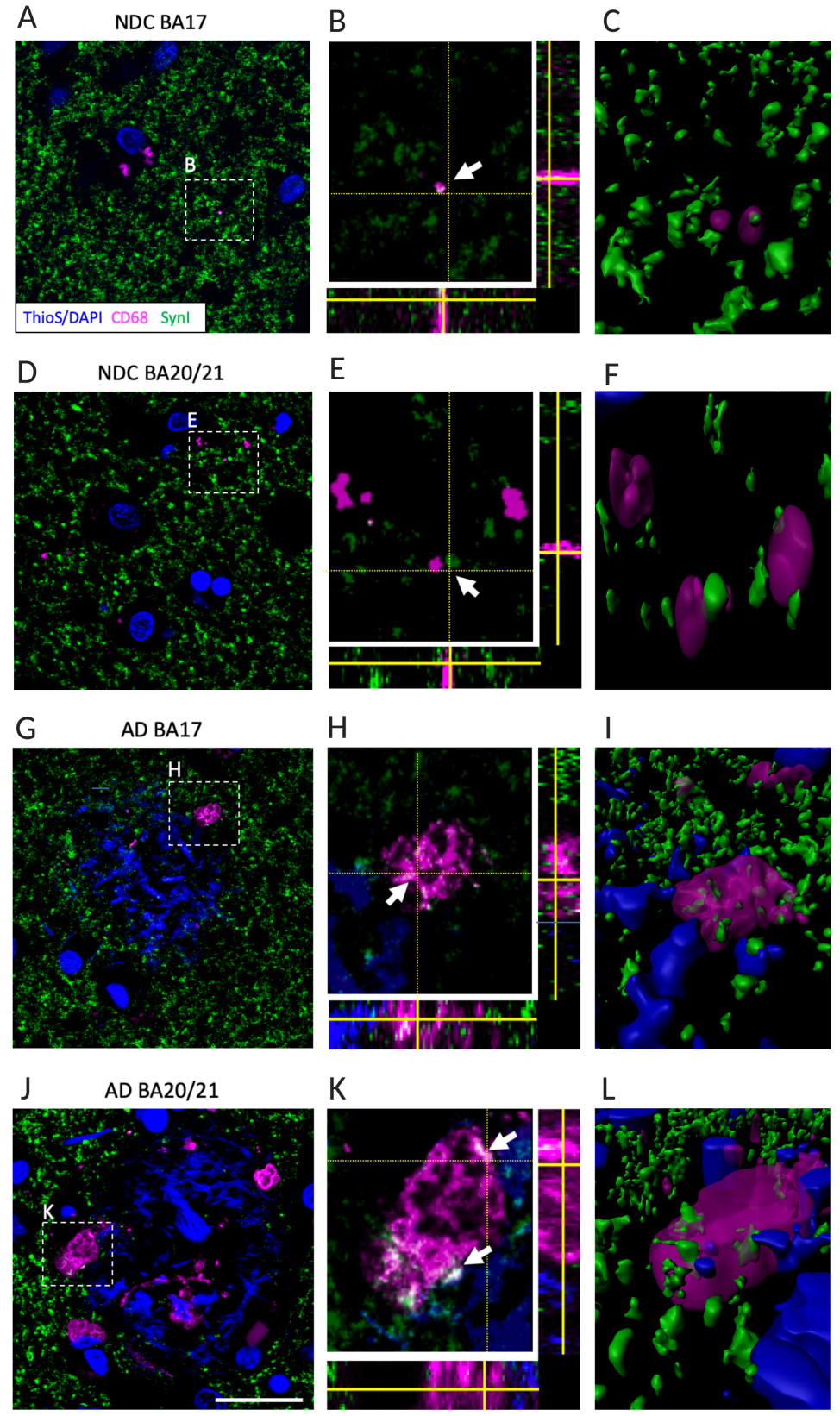

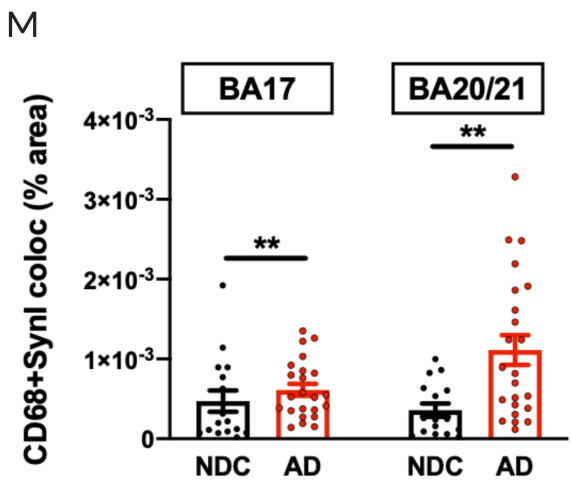

N
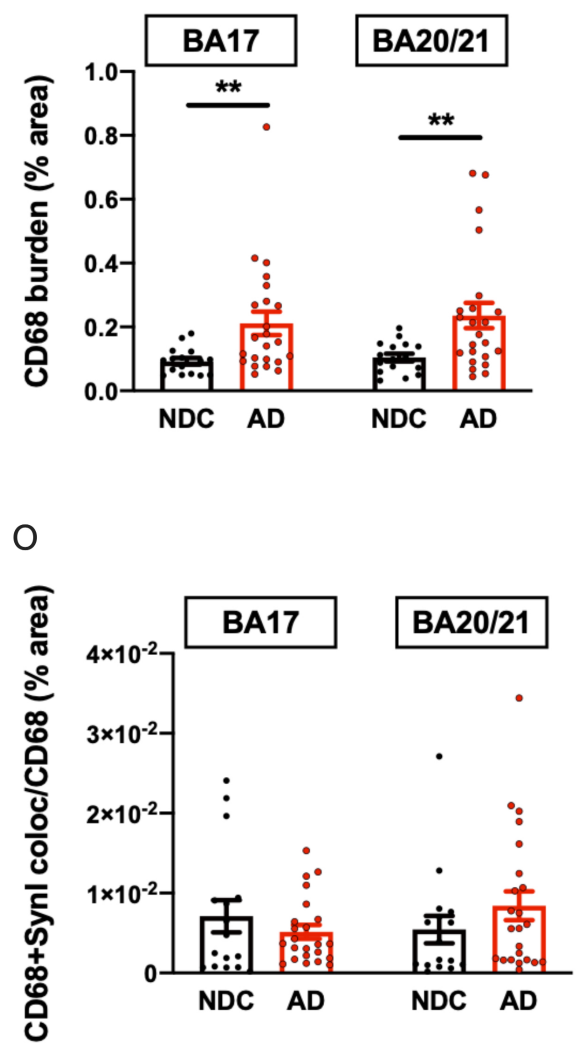

Figure 1. Increased presence of synapsin 1 in CD68-positive microglial cells in Alzheimer's disease. (A, D, G, J) Confocal images reveal SynI staining (green) localised within or contacting CD68 positive microglial lysosomes (magenta) in both Alzheimer's disease (AD, n=23) and nondemented control (NDC, $\mathrm{n}=16$ ) brain (arrows). Nuclei are labelled with DAPI and plaque fibrils with thioflavin $\mathrm{S}$ (blue). Large images in $\mathrm{A}, \mathrm{D}, \mathrm{G}, \mathrm{J}(75 \times 75 \mu \mathrm{m})$ show average projections of a stack of 10 optical sections. (B, E, H, K) Insets $(15 \times 15 \mu \mathrm{m})$ show zoomed-in and orthogonal views of the stacks. (C, F, I, L) 3-D reconstructions using Imaris. Image stacks from BA17 (occipital lobe) and BA20/21 (inferior temporal lobe) were used to calculate the percent area of overlapping CD68 and SynI staining (M), \% CD68 burden (N), and CD68+synapsinI colocalization area normalised to the CD68 burden (O). Data were analysed with a linear mixed effects model on Tukey transformed data to fit the assumptions of the model (untransformed shown here). Analysis 
shows a significant increase in colocalization of CD68 and SynI in AD compared to control (M, post-hoc pairwise comparisons $\mathrm{p}=0.00482$ ). The percent area occupied by CD68 (burden) is significantly increased in AD compared to control cases $(\mathrm{N}$, post-hoc pairwise comparisons $\mathrm{p}=0.00116)$. When the colocalization of CD68+synapsin I is normalised to CD68 burden $(\mathrm{G})$, there are no longer effects of genotype or brain region. Each dot in bar graphs represents the mean of 20 values for a single case, with the bar showing the mean average and error bars representing SEM. For statistics, $* * \mathrm{p}<0.01$. Scale bar represents $20 \mu \mathrm{m}$.

To date, one of the most well established mechanisms for synapse loss in AD is the synaptic accumulation of toxic oligomeric $A \beta$, which is found around senile plaques (24). Furthermore, microglia surround $A \beta$ plaques and upregulate inflammatory and phagocytic markers $(6,25-27)$. Hence, we hypothesised microglia phagocytose more synapses close to $\mathrm{A} \beta$ plaques in $\mathrm{AD}$ cases, contributing to the exacerbated synapse loss near plaques. Indeed, near plaques we observed a marked increase in CD68 and Syn1 colocalisation in both brain areas (Figs. 2A-F, linear mixedeffects model on Tukey transformed data: effect of plaque $\beta=0.05061$, stderr $=0.01370$, $\mathrm{p}=0.00048$ ), but no significant differences between brain regions. Similarly, we observed increased CD68 burden near plaques (Fig. 2G, linear mixed-effects model on Tukey transformed data: effect of plaque $\beta=0.08333$, stderr $=0.009600, p=2.26 \times 10^{-12}$ ). Of note, synaptic internalisation was also evident in regions distant from plaques, suggesting it is not an entirely plaque-dependent phenomenon (Figs. 2B and D). When normalised to CD68 burden, the synaptic colocalization in microglia near plaques was not found to be increased $(\mathrm{p}=0.0704)$ (Fig. $2 \mathrm{H})$, again implicating microgliosis or hypertrophy in increased synaptic ingestion near plaques. 
A

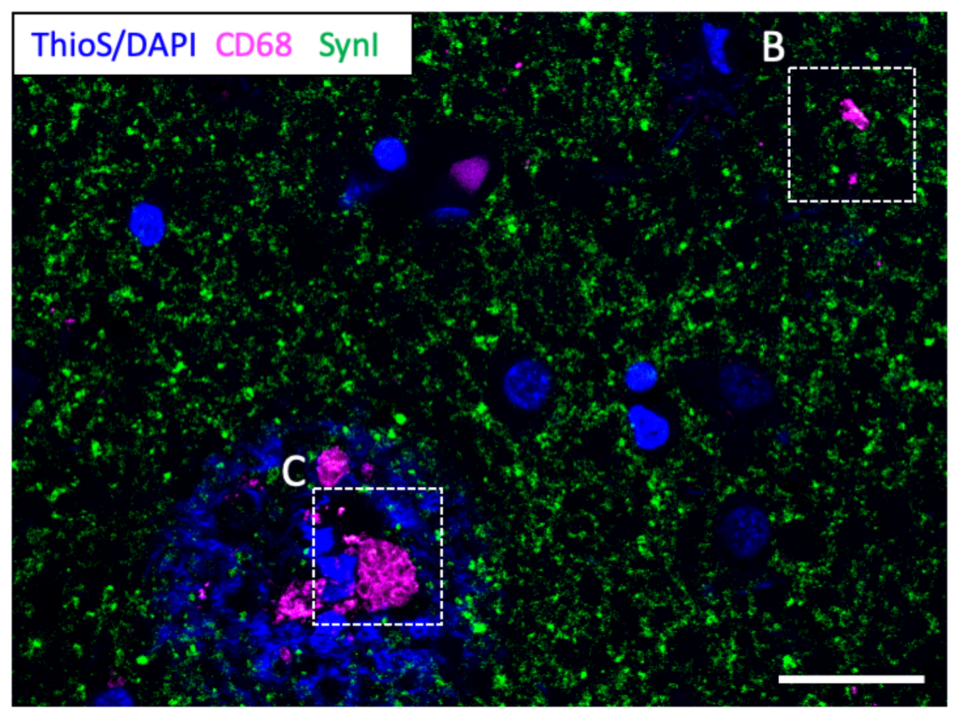

$\mathrm{F}$

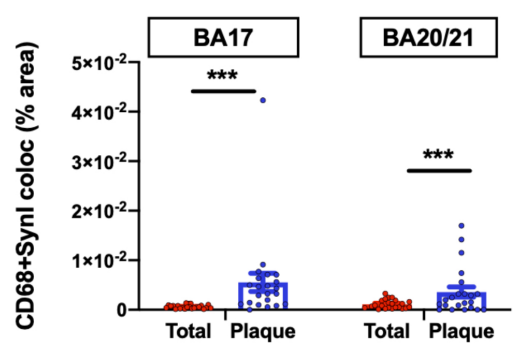

B

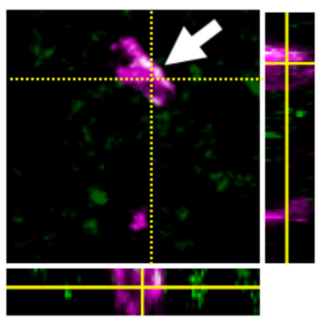

C

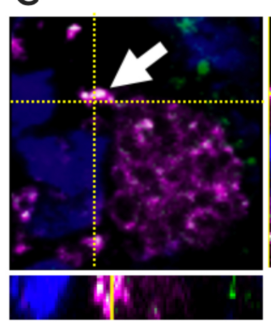

D

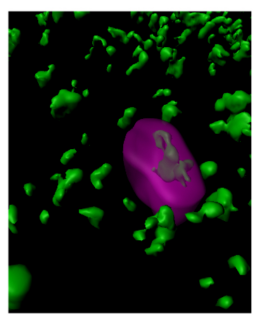

$\mathrm{E}$

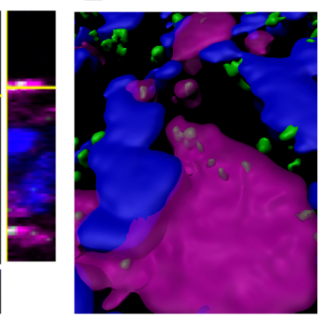

$\mathrm{H}$

Figure 2. Plaque-associated increased synapsin $I$ in CD68-positive microglial cells in Alzheimer's disease. (A) Confocal image (average projections of a stack of 10 optical sections) reveals SynI staining (green) localised within or contacting CD68 positive microglial lysosomes (magenta) in Alzheimer's disease (AD, $n=22)$. Nuclei are labelled with DAPI and plaque fibrils with thioflavin $\mathrm{S}$ (blue). (B and C) Insets $(15 \times 15 \mu \mathrm{m})$ show zoomed-in and orthogonal views of the stacks. (D and E) 3-D reconstructions using Imaris. Image stacks from BA17 (occipital lobe) and BA20/21 (inferior temporal lobe) were used to calculate the percent area of overlapping CD68 and SynI staining (F), \% CD68 burden (G), and CD68+synapsinI colocalization area normalised to the CD68 burden $(\mathrm{H})$. Data were analysed with a linear mixed effects model on Tukey transformed data to fit the assumptions of the model (untransformed shown here). Analysis shows a significant increase in colocalization of CD68 and SynI in AD near plaques compared to total area $(\mathrm{F}$, post-hoc pairwise comparisons $\mathrm{p}=0.0003)$. The percent area occupied by CD68 (burden) is also significantly increased near plaques compared to total area $(\mathrm{G}$, post-hoc pairwise comparisons $\mathrm{p}<0.0001)$. When the colocalization of CD68+SynI is normalised to CD68 burden $(\mathrm{H})$, there are no longer effects of genotype or brain region $(\mathrm{p}=0.0704)$. Each dot in the box and whisker plot represents a single case, with the bar showing the mean average and error bars representing SEM. For statistics, $* * * p<0.001, * * * * p<0.0001$. Scale bar represents $20 \mu \mathrm{m}$. 
Having shown in post-mortem human tissue that there is more synaptic protein located within microglia in $\mathrm{AD}$ compared to control brain, we next sought to determine whether synaptic fractions derived from human AD brain are more readily internalised by microglia in culture. We used fresh frozen samples from the temporal lobe of controls and AD cases to prepare synaptoneurosomes in synapse-enriched fractions (SEFs) (28). The synaptoneurosomes were shown by Western blotting to have significantly higher levels of both synaptophysin (Sy38) and post-synaptic density 95 (PSD-95) in the SEFs compared to total brain homogenate, as well as a significant de-enrichment of the nuclear marker histone H3 (Figs. S2A-E). Interestingly, we observed lower levels of synaptophysin in AD brains compared to brains from age-matched non-demented controls. However, PSD-95 protein levels were unchanged, consistent with literature showing pre-synaptic terminals are more vulnerable in disease (29) (Figs. S2F\&G).

Synaptoneurosomes were conjugated to pHrodo-Red SE, enabling visualisation of synapses internalised within the acidic phago-lysosomal compartment during phagocytosis. Initially, the BV2 murine microglial cell line was used to validate the pHrodo-tagged synaptoneurosomes. Clear uptake of synaptoneurosomes into acidic subcellular compartments was observed (Movies S1\&2) and this was diminished by addition of $10 \mu \mathrm{M}$ cytochalasin $\mathrm{D}(\mathrm{CytD})$, a potent inhibitor of actin polymerisation (Movie S3). Once the assay was established, we tested uptake of synaptoneurosomes from control or AD brains by primary human microglia isolated from nondiseased temporal cortex neurosurgical biopsies. Of note, microglia isolated from surgical resection demonstrated comparable transcriptomic signatures between non-affected cortex and exvivo isolated microglia (30). Human microglia were exposed to AD or control synaptoneurosomes labelled with pHrodo and imaged using live microscopy (31) (Fig. 3A, Movies S4\&5). Synaptoneurosomes from both AD and control brains were rapidly taken up with a peak of $45 \%$ of cells actively phagocytosing by 60 mins (Fig. 3B). Importantly, phagocytosis of synaptoneursomes was massively downregulated in CytD treated cells. When synaptoneurosomes from $\mathrm{AD}$ brains were incubated with microglia, significantly more cells phagocytosed synaptoneurosomes, as shown by the increased area under the curve (Fig. 3C). Moreover, ADderived synaptoneurosomes were phagocytosed more rapidly by human microglia cells compared to controls, indicated by the reduced time to half maximum value for the phagocytotic index (Fig. 3D). When human synaptoneurosomes were incubated with mouse primary microglia (32) we 
observed a similar effect; AD synaptoneurosomes were eaten by more microglia and more rapidly (Figs. S3A-D, Movies S6\&7). Overall, these data suggest that synapses from AD brain are more susceptible to phagocytosis by microglia.

A
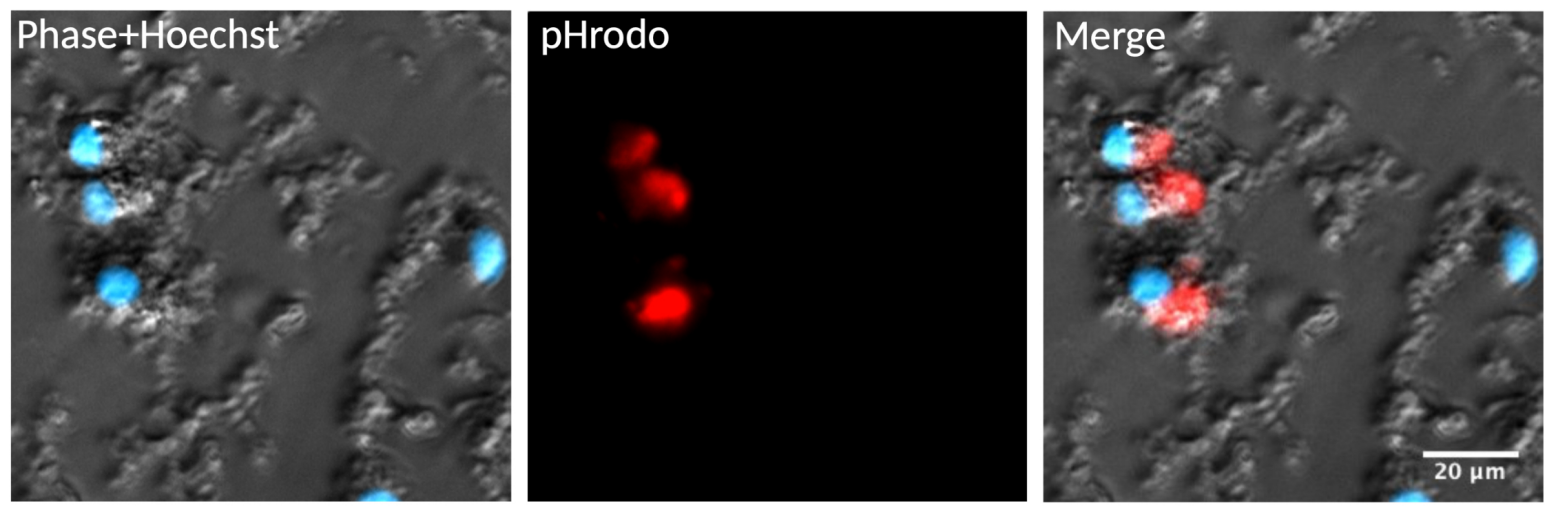

B

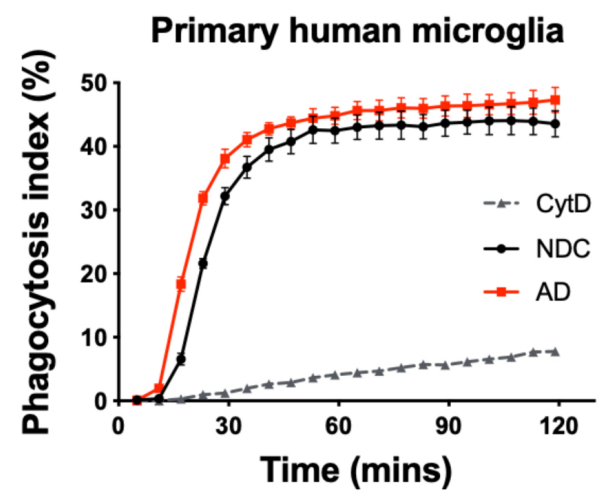

C

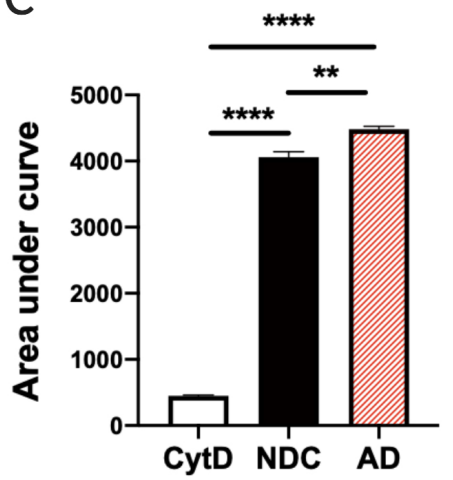

D

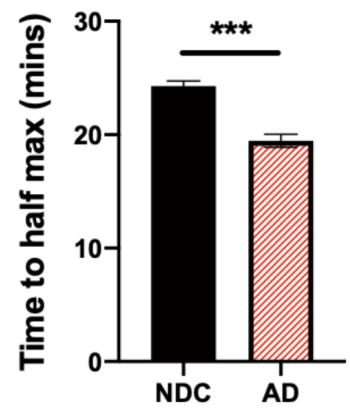

Figure 3. Increased phagocytosis of AD-derived synaptoneurosomes by primary human microglia. (A) Representative images of phagocytosis assay using primary human microglia (grey), from live imaging, showing the phagocytosed pHrodo-tagged synaptoneurosomes (red) from NDC and AD brains and microglial nuclei (cyan). (B) Phagocytosis index of primary human microglia engulfing human synaptoneurosomes ( $\mathrm{n}=1,6$ repeats for NDC and 3 repeats for AD and CytD). CytD was used as negative control by inhibiting phagocytosis. (C) Area under curve from $\mathrm{B}$ showing synaptoneurosomes from $\mathrm{AD}$ cases had a greater area under the curve than both control synaptoneurosome experiments, indicating greater proportion of microglia undergoing phagocytosis (ordinary one-way ANOVA with Tukey's multiple comparisons test, $\mathrm{p}=0.0039$ ). (D) Time to half-maximum phagocytosis was calculated from the curve shown in B, and shows that synaptoneurosomes from $\mathrm{AD}$ cases are phagocytosed quicker than ones from control cases (unpaired Student's t-test, $\mathrm{p}=0.0005$ ). For statistics, ${ }^{* *} \mathrm{p}<0.01,{ }^{* * *} \mathrm{p}<0.001,{ }^{* * * *} \mathrm{p}<0.0001$. Data shown as mean \pm SEM. 
In conclusion, our data show that human microglia ingest synapses and that more uptake occurs in AD than control brain. In AD, this could be beneficial in clearing dysfunctional or dead synapses or could be harmful in contributing to synaptic degeneration and removal of functional synapses. It is likely that both are true with a differing balance of beneficial and harmful contributions of microglia to synaptic phenotypes at different stages of disease and in different parts of the brain. With a clearer understanding of microglial-synapse interactions, there is potential to develop effective therapeutics to protect synapse function. Drugs that attenuate microglial proliferation and anti-complement therapies have shown some potential to reduce AD-associated pathology in mice, and these could be refined based on their potential to help protect synapses in future $(14,15,33)$. Our data address the key knowledge gap in the field of whether adult human microglia phagocytose synaptic proteins. We provide direct evidence from post-mortem brain and live microglia isolated from adult brain that human microglia engulf synaptic proteins and that this is exacerbated in AD. This supports future development of therapeutics targeting microglial interactions with synapses and demonstrates the utility of a culture model feeding human AD brain-derived synapses to cultured microglia to test mechanisms and potential therapeutics. 


\title{
Materials and Methods
}

\author{
Animals \\ Experiments were performed using male C57B1/6J mice (Charles River Laboratories). Mice \\ maintained under a standard $12 \mathrm{~h}$ light/ dark cycle and provided with ad libitum access to food and \\ water. Mice were housed in groups of up to five mice and were acclimatized for a minimum of 1 \\ week prior to procedures. All experiments were conducted under the UK Home Office Animals \\ (Scientific Procedures) Act 1986, in agreement with local ethical and veterinary approval \\ (Biomedical Research Resources, University of Edinburgh).
}

\section{Human tissue}

All tissue was provided by the MRC Edinburgh Brain Bank, following all appropriate ethical approval. For paraffin embedding, tissue was dehydrated via increasing ethanol solutions, fixed in formalin, and baked in paraffin-embedded blocks. Tissue from the inferior temporal lobe (Broadmann area 20/21) and primary visual cortex (Broadmann area 17) was cut using a microtome at $4 \mu \mathrm{m}$ thickness and mounted on glass slides for use in immunohistochemistry. AD cases were cross-checked neuropathologically and were confirmed to be Braak Stages V-VI. In one case (BBN: 24527) no plaques were measured in BA17 and was excluded as a whole from the near plaque analysis. Case BBN31495 has a Braak Stage of VI but has been cognitively tested upon 3 waves and was cognitively unimpaired. Unfixed frozen tissue was provided in 300-500mg blocks. Data about subjects included in the study are found in Supplementary Table 1.

The human post-mortem tissue study plan was published on the Edinburgh DataShare repository https://doi.org/10.7488/ds/2335 (34).

Use of human tissue for post-mortem studies has been reviewed and approved by the Edinburgh Brain Bank ethics committee and the ACCORD medical research ethics committee, AMREC (approval number 15-HV-016; ACCORD is the Academic and Clinical Central Office for Research and Development, a joint office of the University of Edinburgh and NHS Lothian). The Edinburgh Brain Bank is a Medical Research Council funded facility with research ethics committee (REC) approval (11/ES/0022). 
Mean data for each case from human post-mortem tissue studies can be found in supplemental table 2 (total field) and supplemental table 3 (AD cases with plaque data).

\section{Immunohistochemistry}

Paraffin-embedded sections were dewaxed in xylene for 6 minutes, followed by rehydration using descending ethanol-to-water solutions (100\% EtOH, 90\% EtOH, 70\% EtOH, 50\% EtOH, 100\% water). For antigen retrieval, samples were pressure cooked for 3 minutes in citrate buffer, pH 6 (Vector labs, H3300). Slides were incubated with an autofluorescence eliminator reagent (Merck, 2160) for 5 minutes and washed with 70\% EtOH. The tissue was then blocked for 1 hour with $10 \%$ normal donkey serum (Sigma-Aldrich, D96663) and 0.3\% Triton X-100. The following primary antibodies were incubated overnight at $4^{\circ} \mathrm{C}$ in blocking solution: CD68 (mouse monoclonal, 1:100, DAKO M0876), Synapsin I (rabbit, 1:750, Merck Millipore AB1543P), Iba1 (goat polyclonal, 1:500, Abcam ab5076), and TMEM119 (rabbit polyclonal, 1:500, Abcam ab185333). For Thioflavin S (Sigma-Aldrich, T1892), slides were dipped in 0.001\% Thioflavin S, made in 50\% ethanol, for 8 minutes and differentiated in $80 \%$ ethanol for 1 minute. All secondary antibodies (highly cross-adsorbed) were purchased from ThermoFischer Scientific and were used at a concentration of 1:500. Nuclei were counterstained with DAPI $(1 \mu \mathrm{g} / \mathrm{ml})$ (D9542-10MG, SigmaAldrich).

\section{Confocal microscopy}

Slides were imaged on a confocal microscope (Leica TCS8) with a 63x oil immersion objective. Laser and detector settings were kept constant between samples. During imaging and analysis, the experimenter was blinded. Twenty images from the grey matter were taken per case, sampling randomly through all six cortical layers. For analysis, FIJI was used to create a 20-micron halo from the core of $A \beta$ plaques, which was considered as near plaques. Using a custom written macro, images were automatically thresholded to perform a mask of the microglial and synaptic stains, which were multiplied to show the percent area of colocalisation. The twenty images taken per slide were averaged as a single value for each case. 


\section{Synapse-enriched fraction preparation}

Preparation of synaptoneurosomes was performed as described previously (28). Snap-frozen human tissue of 300-500mg from BA38 (temporal cortex) was homogenised using a Dounce homogenizer with $1 \mathrm{ml}$ of a protease inhibitor buffer, termed here Buffer A. Buffer A consists of 25mM HEPES, $120 \mathrm{mM} \mathrm{NaCl}, 5 \mathrm{mM} \mathrm{KCl}, 1 \mathrm{mM} \mathrm{MgCl} 2,2 \mathrm{mM} \mathrm{CaCl}_{2}$, protease inhibitors (Merck, 11836170001) and phosphatase inhibitors (Merck, 524629-1SET) made up in sterile water, and was prepared fresh each day. An initial filtration using an 80-micron filter (Merck, NY8002500) removed debris and yielded the total homogenate $(\mathrm{TH})$. A sample of the TH was snap-frozen on dry ice for Western blot analysis, and the rest was split in half for Western blot analysis and the phagocytosis assays. A subsequent filtration took place using a 5-micron filter (Merck, SLSV025LS) followed by centrifugation at 1000xg for 7 minutes to yield the synaptoneurosome (SN) pellet. The pellet was washed with Buffer A and centrifuged again with the same conditions to ensure purity. Pellets were snap frozen on dry ice and stored at $-80^{\circ} \mathrm{C}$ for long-term storage.

\section{Protein Extraction}

For protein extraction, samples we diluted five-fold with Tris-HCl buffer $\mathrm{pH} 7.6$ (100mM Tris$\mathrm{HCl}, 4 \% \mathrm{SDS}$, and Protease inhibitor cocktail EDTA-free [ThermoFischer Scientific , 78447]), followed by centrifuging for 25 minutes at 13,3000 RPM at $4^{\circ} \mathrm{C}$. Then, the supernatant was collected in fresh tubes and heated at $70^{\circ} \mathrm{C}$ for 10 minutes. Samples were kept at $-80^{\circ} \mathrm{C}$ for longterm storage.

\section{Micro BCA}

Micro BCA kit (ThermoFischer Scientific, 23235) was used to quantify protein levels, following manufacturers instructions. Briefly, $1 \mu \mathrm{l}$ of sample was added in $1 \mathrm{ml}$ of working solution and heated at $60^{\circ} \mathrm{C}$ for 1 hour. Absorbance values were obtained using spectrophotometry at $562 \mathrm{~nm}$ and were compared to a standard curve using specimens of known protein concentration, provided by the kit. 


\section{Western Blot}

After protein extraction, each sample was made-up to 20ug of protein in Lamelli buffer. Samples were run in NuPAGE buffer (ThermoFischer Scientific, NP0002) on 4-12\% Bis-Tris gels (ThermoFischer Scientific, NP0323BOX) for 2 hours at 100V. Each gel was run with a molecular weight marker (Licor, 928-40000) in the first well. Gels were then soaked in 20\% ethanol for 10 minutes prior to transferring using the iBlot 2 transferring system. Pre-packed transfer stacks containing a PVDF membrane (ThermoFischer Scientific, IB24002) were assembled as per manufacturers recommendations, and samples were transferred for 8.5 minutes at $25 \mathrm{~V}$. After transferring, the PVDF membranes were stained for 5 minutes with Ponceau $\mathrm{S}$ in $5 \%$ acetic acid (P7170-1L), and washed 3 times with 5\% acetic acid to stain for total protein. Ponceau S stained membranes were scanned and analysed on ImageJ to obtain a measurement of total protein per sample. Ponceau S was washed out with PBS and the PVDF membranes were blocked using Odyssey blocking buffer (Licor, 927-40000) for 30 minutes, following overnight incubation with primary antibodies made up in Odyssey block with $0.1 \%$ Tween-20, at $4^{\circ} \mathrm{C}$ with gentle shaking. The following primary antibodies were used: Synaptophysin (mouse, Abcam ab8049, 1:1000), PSD-95 (rabbit, Cell signalling D27E11, 1:1000), and Histone (rabbit, Abcam ab1791, 1:1000). The next day, membranes were washed 6 times with PBS-0.1\% Tween-20, and incubated with the following Licor secondary antibodies for 30 minutes: IRDye 680RD Donkey anti-Mouse IgG, highly cross-adsorbed (Licor, 925-68072, 1:5000) and IRDye 800CW Donkey anti-Rabbit IgG, highly cross-adsorbed (Licor, 925-32213, 1:5000). All gels were imaged using the same exposure times and intensities using a Licor Scanner. The images were then uploaded on ImageStudio for analysis. For each band, the same size box was used to ensure all samples are measured equally. Each sample was normalised to its corresponding value of total protein.

\section{Synaptoneurosome labelling with pHrodo Red-SE}

First, pHrodo Red-SE (ThermoFischer Scientific, P36600) was diluted with DMSO according to manufacturers instructions to reach a concentration of $10 \mathrm{mM}$. Synaptoneurosome (SNS) pellets were resuspended in $100 \mathrm{mM}$ sodium carbonate buffer $\mathrm{pH}$ 9, adapted from a previously described protocol (35). SNSs were tagged with pHrodo Red-SE based on protein concentration, roughly at 
for 1 hour, covered in foil. Samples were centrifuged at 13,000 RPM for 10 minutes to obtain the labelled SNS pellet, followed by 3 rounds of washing with PBS and centrifugation to wash out any unbound dye. The pHrodo-labelled SNS pellets were then resuspended in 5\% DMSO-PBS and aliquoted for storage at $-80^{\circ} \mathrm{C}$. Aliquots of pooled AD and NDC samples were prepared fresh.

\section{BV2 microglia phagocytosis assay}

Phagocytosis assays were optimised using the BV2 immortalised murine microglia cell line. BV2 microglia were cultured in DMEM + GlutaMAX (ThermoFischer Scientific, 31966-021) and were supplemented with 10\% fetal bovine serum (FBS, ThermoFisher Scientific) and 1\% penicillin/streptomycin (PenStrep, ThermoFischer Scientific). Cells were grown in a humiditycontrolled incubator at $37^{\circ} \mathrm{C}$ with $5 \% \mathrm{CO}_{2}$. One day prior to the phagocytosis assay, $\mathrm{BV} 2$ microglia were seeded in a 96-well flat bottom plate (ThermoFischer Scientific, 165305) at a density of 12,500 cells/well. Cells were stained with Hoechst $(2 \mu \mathrm{g} / \mathrm{ml}$, ThermoFischer, H3570) to visualise nuclei and cytochalasin D treated cells $(10 \mu \mathrm{M}$, Sigma-Aldrich, C8273) were used as negative controls. BV2 cells were challenged with pHrodo-tagged synaptoneurosomes, and immediately taken to an ImageExpress high-throughput microscope (Molecular Devices) for live-imaging using a 20x air objective $\left(37^{\circ} \mathrm{C}, 5 \% \mathrm{CO}_{2}\right)$. Images were taken every 5-10 minutes for 3 hours, using the same settings for all imaging sessions. All conditions were repeated in triplicate with 4 fields of view per well. For analysis, MetaXpress 6 (Molecular Devices) software was used to automatically calculate the number of cells per field of view and detect fluorescence around cells. A phagocytosis index was calculated by normalising the number of cells phagocytosing to total number of cells in an automated and unbiased way. The same exposure settings were used for each set of experiments. Videos taken with phase contrast (Movies S1, 4 and 6) were recorded on a Zeiss Observer Z1 microscope using $20 \mathrm{x}$ air objective $\left(37^{\circ} \mathrm{C}, 5 \% \mathrm{CO}_{2}\right)$, with images taken every 5 minutes, and were used for Figure 3A and Figure S2A.

\section{Primary mouse microglia}

Primary adult mouse microglia were isolated and cultured as described previously (32). Brains from 12-week-old male C57/B16 (Charles-River) mice were isolated by terminally anaesthetizing 
with $3 \%$ isoflurane $\left(33.3 \% \mathrm{O}_{2}\right.$ and $\left.66.6 \% \mathrm{~N}_{2} \mathrm{O}\right)$ and transcardial perfusion with ice-cold $0.9 \%$ $\mathrm{NaCl}$. Brains were immediately placed into ice-cold HBSS (ThermoFischer Scientific) and minced using a 22A scalpel before centrifugation ( $300 \mathrm{x} \mathrm{g}, 2 \mathrm{~min})$ and digestion using the MACS Neural Dissociation Kit (Miltenyi) according to manufacturer's instructions. Briefly, brain tissue was incubated in enzyme P (50 $\mu \mathrm{L} /$ brain $)$ diluted in buffer $\mathrm{X}(1900 \mu \mathrm{L} /$ brain $)$ for $15 \mathrm{~min}$ at $37^{\circ} \mathrm{C}$ under gentle rotation before addition of enzyme A (10 $\mu \mathrm{L} /$ brain) in buffer Y (20 $\mu \mathrm{L} / \mathrm{brain})$ and further incubation for $20 \mathrm{~min}$ at $37^{\circ} \mathrm{C}$ under gentle rotation. Following digest tissue was dissociated mechanically using a Dounce homogenizer (loose Pestle, 20 passes) on ice and centrifuged (400 x $\mathrm{g}, 5 \mathrm{~min}$ at $4^{\circ} \mathrm{C}$ ). To remove myelin, tissue was resuspended in 35\% isotonic Percoll (GE Healthcare) overlaid with HBSS and centrifuged $\left(800 \mathrm{xg}, 40 \mathrm{~min}, 4^{\circ} \mathrm{C}\right)$. Following centrifugation, the supernatant and myelin layers were discarded and the pellet resuspended in MACS buffer (PBS, 0.5\% low endotoxin BSA (Sigma-Aldrich), $2 \mathrm{mM}$ EDTA, $90 \mu \mathrm{L} / \mathrm{brain}$ ). Anti-CD11b microbeads (Miltneyi) were added $\left(10 \mu \mathrm{L} /\right.$ brain) and the suspension incubated for 15 min at $4{ }^{\circ} \mathrm{C}$. before running through pre-rinsed (MACS buffer) LS columns attached to a magnet (Miltenyi). After washing with $12 \mathrm{~mL}$ MACS buffer columns were removed from the magnet and cells retained (microglia) were flushed in $5 \mathrm{~mL}$ MACS buffer. Microglia were resuspended in DMEM/F-12 (ThermoFischer Scientific) supplemented with 1\% PenStrep, 10\% FBS, 500 pg/mL rhTGF $\beta$-1 (Miltenyi), $10 \mathrm{pg} / \mu \mathrm{L}$ mCSF1 (R\&D Systems). Microglia were counted using a haemocytometer and plated out at 40,000 cells/well onto a black-walled, optical bottom 96-well plate (ThermoFischer Scientific) coated with poly-L-lysine. Cells were cultured for 7 days with a half media change on day 3. Phagocytosis assay was performed and analysed as described above with 9 fields of view per well.

\section{Primary human microglia isolation}

Use of human temporal lobe resections for research was approved by National Health Service Lothian under protocol number 2017/0125/SR/720 issued to V.E.M. The protocol for isolating primary human microglia was adapted from a previously established one (31). Fresh brain tissue was donated from a 23-year-old male undergoing epilepsy surgery. Resected brain specimen came from healthy tissue of the temporal lobe. Briefly, blood was removed by multiple washes of PBS followed by treatment with $0.25 \%$ trypsin and $100 \mathrm{ug} / \mathrm{ml}$ DNAse in PBS for 30 minutes at $37^{\circ} \mathrm{C}$, 
with gentle rotation. Trypsin was then deactivated with $10 \%$ FCS. Samples were centrifuged at 1,200 RPM for 10 minutes (high brakes) at $4^{\circ} \mathrm{C}$ and the supernatant was discarded followed by addition of PBS and Percoll (GE Healthcare) with further ultra-centrifugation at 15,000 RPM for 30 minutes at $4^{\circ} \mathrm{C}$ (no brakes). The myelin layer was aspirated off and cell layer was transferred in a clean tube, leaving behind a layer of red blood cells. The transferred cells were topped-up with PBS and centrifuged again at 1,200 RPM for 10 minutes (high brakes) at $4^{\circ} \mathrm{C}$ and resuspended in warm media containing 5\% FCS and $0.1 \%$ glucose. Subsequently, isolated mixed cells were cultured in T12.5 flasks for 3 days. Three days later, microglia were trypsinised and collected from the flasks, following centrifugation to yield the microglia cell pellet. After the isolation, microglia were counted using a haemocytometer and plated out at 40,000 cells/well onto a black-walled, optical bottom 96-well plate (ThermoFischer Scientific) coated with poly-L-lysine, and were allowed to rest for 5 days prior to phagocytosis assays. Phagocytosis assay was performed and analysed as described above with 9 fields of view per well.

\section{Statistics}

Statistical analyses were performed in Graphpad Prism and R Studio (36). For human post-mortem tissue imaging, data were not normally distributed according to QQ plots. CD68+Syn1 colocalisation data were transformed with a Tukey transformation after adding 1 to all datapoints (lambda value -1082) for normalisation. We used a linear mixed-effects model with disease, APOE genotype, brain region, and sex as fixed effects and case as a random effect to account for multiple measurements per case. Post-hoc comparisons of groups were done using pairwise comparisons in the emmeans function of R. Data is presented in the figures in its untransformed format to allow for scientific interpretation. For all other comparisons, analysis was done GraphPad Prism 8 and appropriate statistical tests were chosen on the basis of normality. In all experiments, for significance we considered $\mathrm{p}<0.05$.

\section{Software and Data Availability}

Figures were made using BioRender, GraphPad Prism, and R Studio. Data from human postmortem tissue imaging is included in supplemental table. The image $\mathrm{J}$ macro used for image 
bioRxiv preprint doi: https://doi.org/10.1101/795930; this version posted October 7, 2019. The copyright holder for this preprint (which was not certified by peer review) is the author/funder, who has granted bioRxiv a license to display the preprint in perpetuity. It is made available under aCC-BY 4.0 International license.

analysis, R Studio scripts used for statistical analyses, raw images used for analysis, and raw movies from cell culture studies are available upon request to the corresponding author. 


\section{References and Notes:}

1. T. L. Spires-Jones, B. T. Hyman, The intersection of amyloid beta and tau at synapses in Alzheimer's disease. Neuron. 82, 756-771 (2014).

2. S. T. DeKosky, S. W. Scheff, Synapse loss in frontal cortex biopsies in Alzheimer's disease: correlation with cognitive severity. Ann. Neurol. 27, 457-464 (1990).

3. R. D. Terry et al., Physical basis of cognitive alterations in Alzheimer's disease: synapse loss is the major correlate of cognitive impairment. Ann. Neurol. 30, 572-580 (1991).

4. T. Minett et al., Microglial immunophenotype in dementia with Alzheimer's pathology. $J$. Neuroinflammation. 13, 135 (2016).

5. $\quad$ P. L. McGeer, E. G. McGeer, Glial cell reactions in neurodegenerative diseases: pathophysiology and therapeutic interventions. Alzheimer Dis Assoc Disord. 12 Suppl 2 , S1-6 (1998).

6. S. Itagaki, P. L. McGeer, H. Akiyama, S. Zhu, D. Selkoe, Relationship of microglia and astrocytes to amyloid deposits of Alzheimer disease. J. Neuroimmunol. 24, 173-182 (1989).

7. T. Jonsson et al., Variant of TREM2 associated with the risk of Alzheimer's disease. $N$. Engl. J. Med. 368, 107-116 (2013).

8. M. C. Chartier-Harlin et al., Early-onset Alzheimer's disease caused by mutations at codon 717 of the beta-amyloid precursor protein gene. Nature. 353, 844-846 (1991).

9. C. M. Karch, A. M. Goate, Alzheimer's disease risk genes and mechanisms of disease pathogenesis. Biol. Psychiatry. 77, 43-51 (2015).

10. D. P. Schafer et al., Microglia sculpt postnatal neural circuits in an activity and complement-dependent manner. Neuron. 74, 691-705 (2012).

11. B. Stevens et al., The classical complement cascade mediates CNS synapse elimination. Cell. 131, 1164-1178 (2007).

12. R. C. Paolicelli et al., Synaptic pruning by microglia is necessary for normal brain development. Science. 333, 1456-1458 (2011).

13. L. Weinhard et al., Microglia remodel synapses by presynaptic trogocytosis and spine head filopodia induction. Nat. Commun. 9, 1228 (2018).

14. S. Hong et al., Complement and microglia mediate early synapse loss in Alzheimer mouse models. Science. 352, 712-716 (2016).

15. Q. Shi et al., Complement $\mathrm{C} 3$ deficiency protects against neurodegeneration in aged plaque-rich APP/PS1 mice. Sci. Transl. Med. 9 (2017), doi:10.1126/scitranslmed.aaf6295.

16. B. Dejanovic et al., Changes in the Synaptic Proteome in Tauopathy and Rescue of TauInduced Synapse Loss by C1q Antibodies. Neuron. 100, 1322-1336.e7 (2018).

17. T. Wu et al., Complement $\mathrm{C} 3$ is activated in human $\mathrm{AD}$ brain and is required for neurodegeneration in mouse models of amyloidosis and tauopathy. Cell Rep. 28, 21112123.e6 (2019).

18. C. M. Henstridge, M. Tzioras, R. C. Paolicelli, Glial contribution to excitatory and inhibitory synapse loss in neurodegeneration. Front. Cell Neurosci. 13, 63 (2019).

19. J. N. Keller, Age-related neuropathology, cognitive decline, and Alzheimer's disease. Ageing Res Rev. 5, 1-13 (2006).

20. M. L. Bennett et al., New tools for studying microglia in the mouse and human CNS. Proc. 
Natl. Acad. Sci. USA. 113, E1738-46 (2016).

21. M. Tzioras, C. Davies, A. Newman, R. Jackson, T. Spires-Jones, Invited Review: APOE at the interface of inflammation, neurodegeneration and pathological protein spread in Alzheimer's disease. Neuropathol Appl Neurobiol. 45, 327-346 (2019).

22. B. Winblad et al., Defeating Alzheimer's disease and other dementias: a priority for European science and society. Lancet Neurol. 15, 455-532 (2016).

23. P. L. McGeer et al., Microglia in degenerative neurological disease. Glia. 7, 84-92 (1993).

24. R. M. Koffie et al., Apolipoprotein E4 effects in Alzheimer's disease are mediated by synaptotoxic oligomeric amyloid- $\beta$. Brain. 135, 2155-2168 (2012).

25. H. Keren-Shaul et al., A Unique Microglia Type Associated with Restricting Development of Alzheimer's Disease. Cell. 169, 1276-1290.e17 (2017).

26. G. A. Rodriguez, L. M. Tai, M. J. LaDu, G. W. Rebeck, Human APOE4 increases microglia reactivity at $\mathrm{A} \beta$ plaques in a mouse model of $\mathrm{A} \beta$ deposition. $J$. Neuroinflammation. 11, 111 (2014).

27. S. Krasemann et al., The TREM2-APOE Pathway Drives the Transcriptional Phenotype of Dysfunctional Microglia in Neurodegenerative Diseases. Immunity. 47, 566-581.e9 (2017).

28. H.-C. Tai et al., Frequent and symmetric deposition of misfolded tau oligomers within presynaptic and postsynaptic terminals in Alzheimer's disease. Acta Neuropathol. Commun. 2, 146 (2014).

29. O. Sheppard, M. P. Coleman, C. S. Durrant, Lipopolysaccharide-induced neuroinflammation induces presynaptic disruption through a direct action on brain tissue involving microglia-derived interleukin 1 beta. J. Neuroinflammation. 16, 106 (2019).

30. T. F. Galatro et al., Transcriptomic analysis of purified human cortical microglia reveals age-associated changes. Nat. Neurosci. 20, 1162-1171 (2017).

31. M. Blain et al., in Protocols for neural cell culture, L. C. Doering, Ed. (Humana Press, Totowa, NJ, 2010), Springer Protocols Handbooks, pp. 87-104.

32. K. Grabert, B. W. McColl, Isolation and phenotyping of adult mouse microglial cells. Methods Mol. Biol. 1784, 77-86 (2018).

33. A. Olmos-Alonso et al., Pharmacological targeting of CSF1R inhibits microglial proliferation and prevents the progression of Alzheimer's-like pathology. Brain. 139, 891907 (2016).

34. M. Tzioras, T. L. Spires-Jones, Research Plan - The effect of APOE genotype on microglial-mediated synapse loss in AD. University of Edinburgh (2018), doi:10.7488/ds/2335.

35. Y. G. Byun, W.-S. Chung, A Novel In Vitro Live-imaging Assay of Astrocyte-mediated Phagocytosis Using pH Indicator-conjugated Synaptosomes. J. Vis. Exp. (2018), doi:10.3791/56647.

36. R. C. Team, R: A language and environment for statistical computing. R Foundation for Statistical Computing, Vienna, Austria. 2012. URL http://www. R. 
Acknowledgments: We wish to thank our human tissue donors and their families for making this work possible. We gratefully acknowledge Edinburgh Neuroscience and Dr Jane Haley MBE for facilitating collaborations leading to this work. We also wish to thank the MRC Edinburgh Brain Bank for providing human tissue. Funding: This work was funded by the UK Dementia Research Institute which receives its funding from DRI Ltd, funded by the UK Medical Research Council, Alzheimer's Society, and Alzheimer's Research UK, the European Research Council (ERC) under the European Union's Horizon 2020 research and innovation programme (Grant agreement No. 681181), Alzheimer's Research UK and the Scottish Government Chief Scientist Office (ARUK SPG2013-1), a Wellcome Trust-University of Edinburgh Institutional Strategic Support Fund, MND Scotland, and Alzheimer's Society. Author contributions: MT, CH, BM, MJDD, and TS$\mathrm{J}$ designed the experiments; MT, MJDD, and CL performed phagocytosis assay experiments; MT and KP stained and analysed human PM tissue; MT and DK made synaptoneurosome preps and performed WB analysis; DS, JK, RKH, and VEM provided human microglia; CS provided human PM tissue; JT and JR assisted with technical help; MT, AS, MJDD, and TSJ handled statistical analysis of data; MT and TS-J wrote the manuscript. All authors contributed in editing the manuscript and providing feedback; Competing interests: TS-J is on the Scientific Advisory Board of Cognition Therapeutics and receives collaborative grant funding from 2 industry partners. None of these had any influence over the current paper. Remaining authors declare no competing interests. Data and materials availability: Data spreadsheets are included as Tables S2. Custom written code used for the analyses are available upon request. Human tissue and cell lines are available subject to ethical approvals and in the case of cell lines, MTAs. 


\section{List of Supplementary Materials:}

A
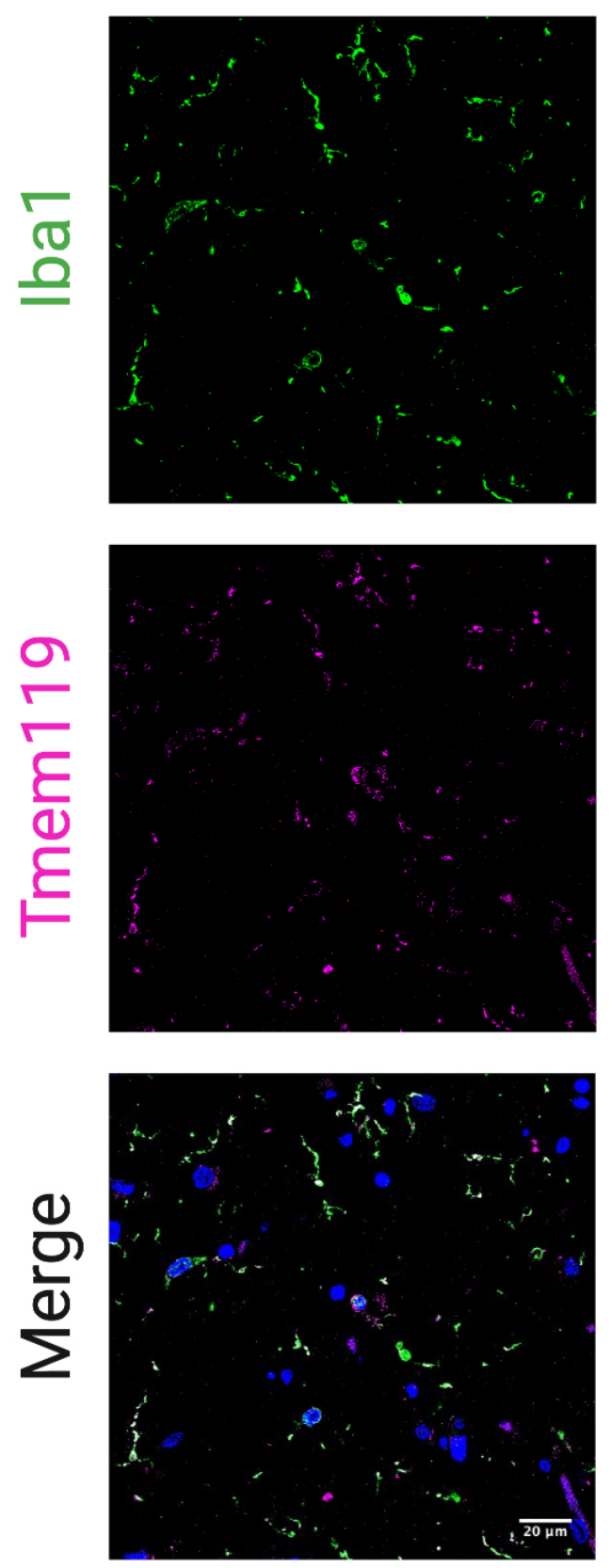

B
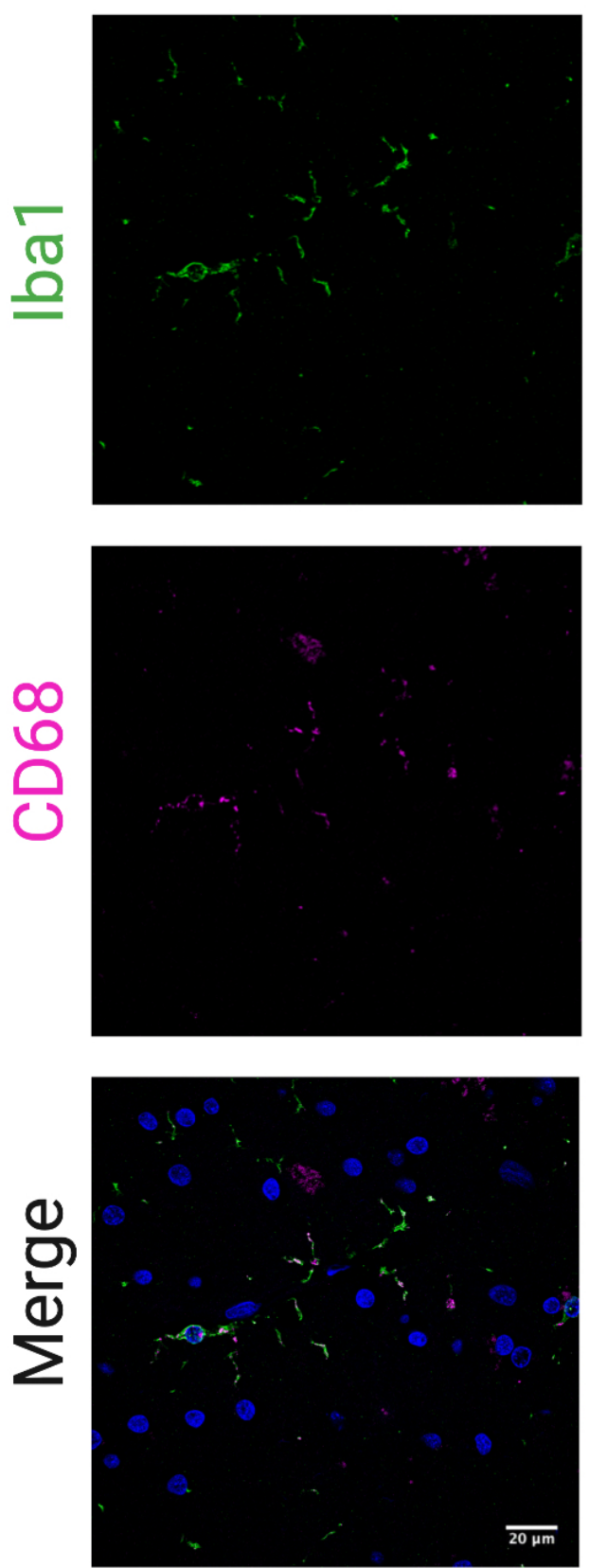

Figure S1. Validation of microglial markers in human post-mortem tissue. Representative confocal images acquired using a $63 x$ oil-immersion lens. Colocalisation of IBA1 (green) with: (A) the microglia-specific marker TMEM119 (magenta), and (B) lysosomal marker CD68 (magenta) inside Ibal positive cells. Nuclei counterstained with DAPI (blue). 
A

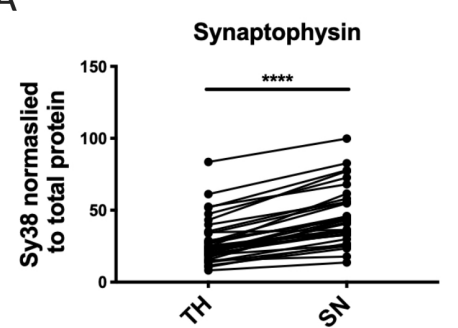

B

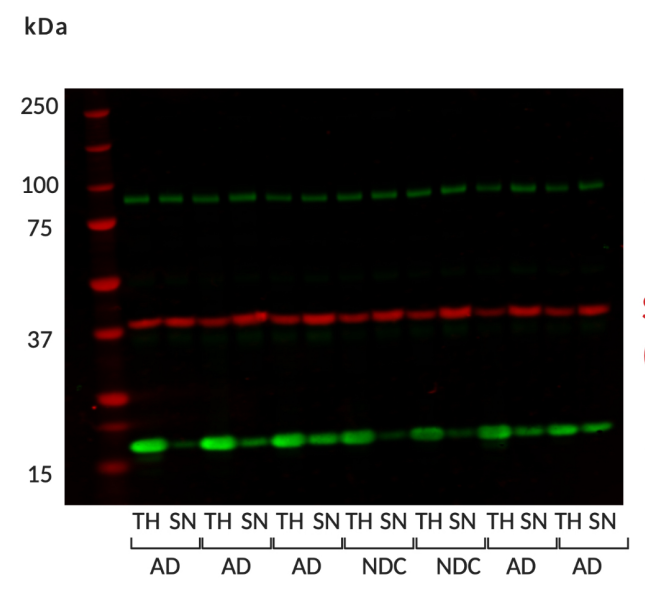

D

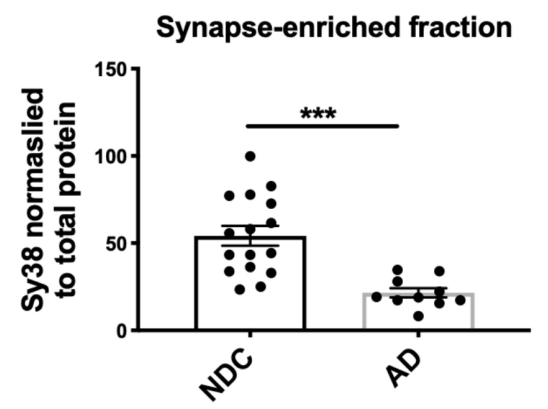

PSD-95

Synaptophysin

(Sy38)

Histone H3

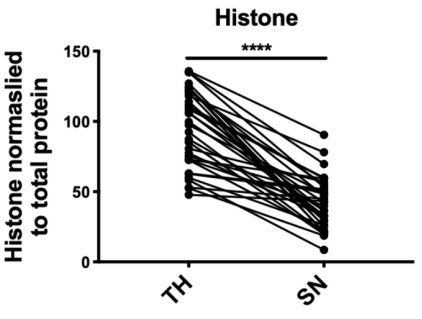

C

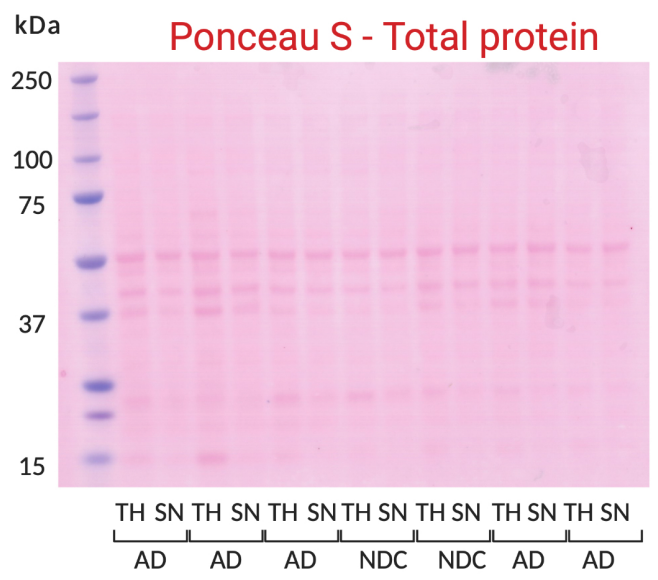

$\mathrm{E}$

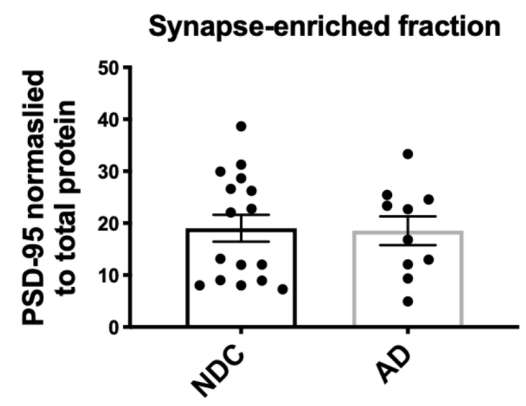

Figure S2. Validation of synaptoneurosome preparations and reduced pre-synaptic protein levels in AD. (A) Significantly increased protein levels of the pre- and post-synaptic markers synaptophysin and PSD-95, respectively, as well as decreased protein levels of histone, indicating exclusion of non-synaptic material (Wilcoxon matched-pairs signed rank test, $* * * * p<0.0001$, $\mathrm{n}=26$ ). Bands were quantified on Image Studio, and normalized to total protein, quantified by Ponceau S. (B) Representative image of Western blot, indicating whether a sample is from total homogenate $(\mathrm{TH})$ or synaptoneurosome $(\mathrm{SN})$, disease status and their respective brain bank number. (C) Ponceau S staining for total protein from gel shown in B. (D) Decreased levels of synaptophysin in AD cases $(n=10)$, compared to age-matched NDC cases $(n=16)$, as detected by Western blot (parametric unpaired Student's t-test, $\mathrm{p}=0.0002$ ). (E) Unchanged PSD-95 protein levels between AD and NDC groups. For statistics, $* * * p<0.001, * * * * p<0.0001$. 
A
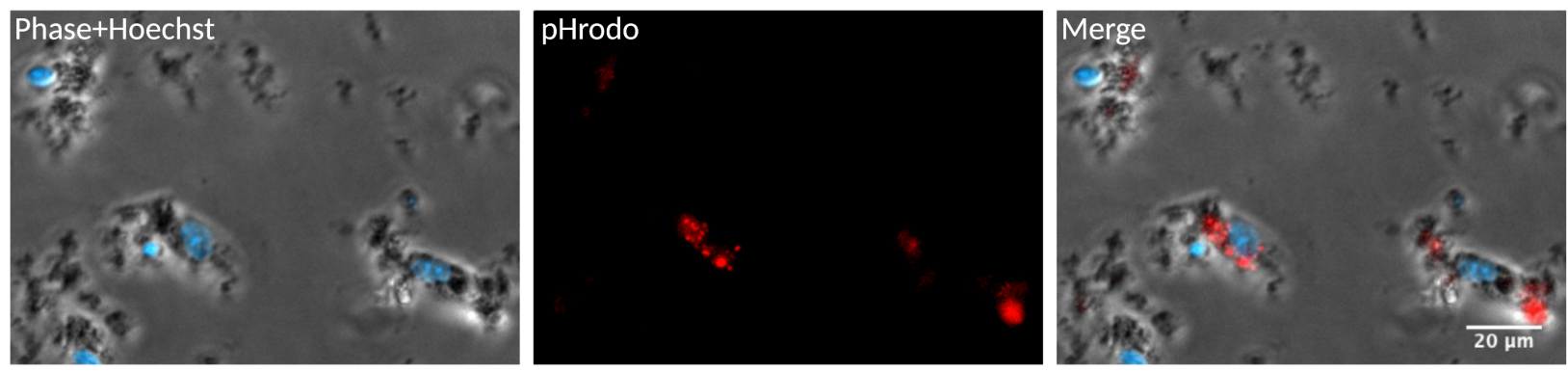

B

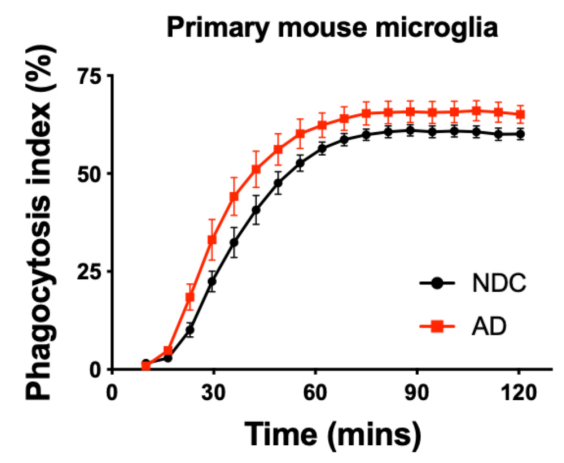

C

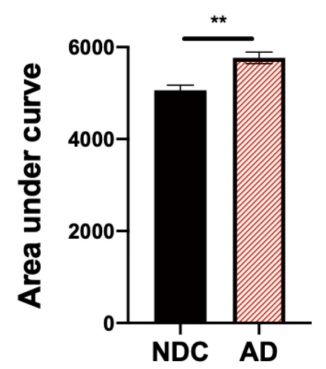

D

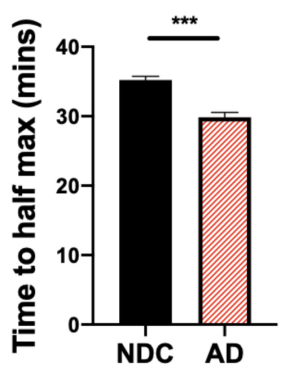

Figure S3. Increased phagocytosis of human synaptoneurosomes by murine primary microglia. (A) Representative images of phagocytosis assay using primary human microglia (grey), from live imaging, showing the phagocytosed pHrodo-tagged synaptoneurosomes (red) from NDC and AD brains and microglial nuclei (cyan). (B) Phagocytosis index of primary mouse microglia engulfing human synaptoneurosomes $(n=4)$. (C) Area under curve from B showing synaptoneurosomes from AD cases had a greater AUC than both control synaptoneurosome experiments (unpaired Student's t-test, $\mathrm{p}=0.0057$ ). (D) Time to half-maximum phagocytosis was calculated from the curve shown in $\mathrm{B}$, and indicates that synaptoneurosomes from $\mathrm{AD}$ cases are phagocytosed quicker than ones from control cases (unpaired Student's t-test, $\mathrm{p}=0.0001$ ). For statistics, $* * \mathrm{p}<0.01, * * * \mathrm{p}<0.001$. Data shown as mean \pm SEM. 


\begin{tabular}{|c|c|c|c|c|c|c|c|c|}
\hline MRC BBN & APOE status (3vs4) & Condition & Age & Gender (F/M) & PMI (hours) & Brain weight $(\mathrm{g})$ & Brain pH & Braak stage \\
\hline $19686^{*}$ & $3 / 3$ & NDC & 76 & \begin{tabular}{|c|}
$\mathrm{F}$ \\
\end{tabular} & \begin{tabular}{|l|}
75 \\
\end{tabular} & 1320 & 6.5 & 1 \\
\hline $28797^{*}$ & $3 / 3$ & NDC & 79 & $\mathrm{M}$ & 57 & 1301 & 6.11 & NA \\
\hline $28406^{*}$ & $3 / 3$ & NDC & 79 & $M$ & 72 & 1437 & 6.13 & II \\
\hline $28402^{*}$ & $3 / 3$ & NDC & 78 & $M$ & 49 & 1503 & 6.33 & 1 \\
\hline $26495^{*}$ & $3 / 3$ & NDC & 78 & $M$ & 39 & 1290 & 6.17 & 1 \\
\hline $14395^{*}$ & $3 / 3$ & NDC & 74 & $\mathrm{~F}$ & 41 & 1520 & 6.3 & NA \\
\hline $20122^{*}$ & $3 / 3$ & NDC & 59 & $M$ & 74 & 1500 & 6.1 & NA \\
\hline $22612^{*}$ & $3 / 3$ & NDC & 61 & $M$ & 70 & 1300 & 6.1 & NA \\
\hline 001.32577 & $3 / 3$ & NDC & 81 & $M$ & 74 & 1313 & 6.07 & NA \\
\hline $29086 * *$ & $3 / 3$ & NDC & 79 & $\mathrm{~F}$ & 68 & 1468 & 6.2 & NA \\
\hline $29082^{*}$ & $3 / 4$ & NDC & 79 & $\mathrm{~F}$ & 80 & 1339 & 5.96 & III \\
\hline $31495^{*}$ & $3 / 4$ & NDC & 81 & $M$ & 38 & 1318 & 5.79 & VI \\
\hline 20593* & $3 / 4$ & NDC & 60 & $M$ & 52 & 1460 & 6 & NA \\
\hline $22629 *$ & $3 / 4$ & NDC & 59 & $\mathrm{~F}$ & 53 & 1280 & 6.3 & NA \\
\hline 15809* & $3 / 4$ & NDC & 58 & $M$ & 90 & 1470 & 5.9 & NA \\
\hline $16425^{*}$ & $3 / 4$ & NDC & 61 & $\mathrm{M}$ & 99 & 1270 & 6.2 & NA \\
\hline 001.34131 & $3 / 4$ & NDC & 82 & $M$ & 95 & 1472 & 5.97 & IV \\
\hline Group mean & $10 / 7$ & & 72.00 & $5 / 12$ & 66.24 & 1385.94 & 6.13 & \\
\hline 24527 & $3 / 3$ & $A D$ & 81 & $\mathrm{M}$ & 74 & 1160 & 6.1 & $\mathrm{~V}$ \\
\hline 28410 & $3 / 3$ & $A D$ & 62 & $\mathrm{~F}$ & 109 & 1029 & 6.04 & $\mathrm{VI}$ \\
\hline 28771 & $3 / 3$ & $A D$ & 85 & $M$ & 91 & 1183 & 5.95 & $\mathrm{VI}$ \\
\hline 15258 & $3 / 3$ & $A D$ & 65 & $M$ & 80 & 1335 & 6.1 & VI \\
\hline 19595 & $3 / 3$ & $A D$ & 87 & M & 58 & 1420 & 6.5 & $\mathrm{VI}$ \\
\hline 19994 & $3 / 3$ & $A D$ & 87 & $\mathrm{~F}$ & 89 & 1270 & 5.9 & VI \\
\hline 22223 & $3 / 3$ & $A D$ & 87 & $\mathrm{~F}$ & 83 & 1200 & 6.7 & IV \\
\hline 001.32929 & $3 / 3$ & $A D$ & 85 & $\mathrm{~F}$ & 80 & 1354 & 6.08 & $\mathrm{VI}$ \\
\hline 19690* & $3 / 4$ & $A D$ & 57 & $M$ & 58 & 1200 & 5.9 & $\mathrm{VI}$ \\
\hline $24322^{*}$ & $3 / 4$ & $A D$ & 80 & $M$ & 101 & 1410 & 6 & VI \\
\hline $25739 *$ & $3 / 4$ & $A D$ & 85 & $\mathrm{~F}$ & 45 & 1375 & 5.77 & VI \\
\hline $26718^{*}$ & $3 / 4$ & $A D$ & 78 & $M$ & 74 & 1367 & 6.13 & $\mathrm{VI}$ \\
\hline 29521* & $3 / 4$ & $A D$ & 95 & $M$ & 96 & 1221 & 6.08 & $\mathrm{VI}$ \\
\hline $29695^{*}$ & $3 / 4$ & $A D$ & 86 & $M$ & 72 & 1200 & 6.1 & VI \\
\hline 10591* & $3 / 4$ & $A D$ & 86 & $\mathrm{M}$ & 76 & 1470 & NA & $\mathrm{VI}$ \\
\hline 15810 & $4 / 4$ & $\mathrm{AD}$ & 73 & $\mathrm{~F}$ & 96 & 1090 & 6.2 & $\mathrm{VI}$ \\
\hline 15811 & $3 / 4$ & $A D$ & 81 & $\mathrm{~F}$ & 41 & 1457 & 6.3 & $\mathrm{VI}$ \\
\hline 23394* & $3 / 4$ & $A D$ & 88 & $\mathrm{~F}$ & 59 & 1165 & 6.3 & $\mathrm{~V}$ \\
\hline $001.26732^{*}$ & $3 / 4$ & $A D$ & 76 & $M$ & 66 & 1467 & 6.48 & VI \\
\hline 20995* & $4 / 4$ & $A D$ & 60 & $M$ & 86 & 1244 & 5.9 & $\mathrm{VI}$ \\
\hline 001.26500 & $4 / 4$ & $A D$ & 81 & $M$ & 83 & 1315 & 6.25 & $\mathrm{VI}$ \\
\hline 15256 & $4 / 4$ & $A D$ & 60 & $M$ & 28 & 1389 & NA & $\mathrm{V}-\mathrm{VI}$ \\
\hline 001.28796 & $4 / 4$ & $A D$ & 60 & $\mathrm{~F}$ & 54 & 1150 & 5.95 & $\mathrm{VI}$ \\
\hline Group mean & $8 / 15$ & & 77.6086957 & $9 / 14$ & 73.86956522 & 1281.347826 & 6.13 & \\
\hline \multicolumn{3}{|c|}{ *denotes cases used for both staaining study and synaptoneurosome preps } & & & & & & \\
\hline \multicolumn{3}{|c|}{ 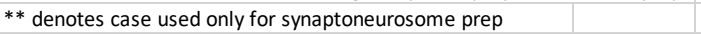 } & & & & & & \\
\hline
\end{tabular}

Table S1. Participant information used for post mortem experiments. For the study, we included in total 17 non-demented control cases (NDC) and 23 Alzheimer's disease (AD) cases, that are age-matched and of mixed APOE genotypes. Age-matching was based on Student's t-test, where $p=0.1064$. All AD cases are end-stage of disease. Some cases were only used for the staining study (no asterisk), some for both the staining and making synaptoneurosomes $(*)$, and one case for only making synaptoneurosomes $(* *)$. NA denotes information is not available. 
Movie S1. BV2 microglia phagocytosis assay with phase contrast. Live imaging of BV2 microglia (phase with Hoechst-positive nuclei in cyan) undergoing phagocytosis of human synaptoneurosomes tagged with pHrodo (can be seen as small spheroids on phase contrast). Synaptoneurosomes become red once they enter the acidic phago-lysosomal compartment of the cell.

Movie S2. BV2 microglia phagocytosis assay. Live imaging of BV2 microglia (Hoechstpositive nuclei in grey) undergoing phagocytosis of human synaptoneurosomes tagged with pHrodo. Synaptoneurosomes can be seen in red as they enter the acidic phago-lysosomal compartment of the cell.

Movie S3. BV2 microglia phagocytosis assay with cytochalasin D. Live imaging of BV2 microglia (Hoechst-positive nuclei in grey) undergoing phagocytosis of human synaptoneurosomes tagged with pHrodo. Cells were treated with $10 \mathrm{uM}$ of Cytochalasin D 30 minutes prior to the experiment to completely inhibit actin polymerization, and by extention block phagocytosis. Synaptoneurosomes can be seen in red as they enter the acidic phagolysosomal compartment of the cell. Phagocytosis is massively decreased as compared to Movie S2.

Movie S4. Primary human microglia phagocytosis assay with phase contrast. Live imaging of primary human microglia from the temporal lobe (phase with Hoechst-positive nuclei in cyan) undergoing phagocytosis of human synaptoneurosomes tagged with pHrodo (can be seen as small spheroids on phase contrast). Synaptoneurosomes become red once they enter the acidic phago-lysosomal compartment of the cell.

Movie S5. Primary human microglia phagocytosis assay. Live imaging of primary human microglia (Hoechst-positive nuclei in grey) undergoing phagocytosis of human synaptoneurosomes tagged with pHrodo. Synaptoneurosomes can be seen in red as they enter the acidic phago-lysosomal compartment of the cell.

Movie S6. Primary mouse microglia phagocytosis assay with phase contrast. Live imaging of primary mouse microglia from the temporal lobe (phase with Hoechst-positive nuclei in cyan) undergoing phagocytosis of human synaptoneurosomes tagged with pHrodo (can be seen as small spheroids on phase contrast). Synaptoneurosomes become red once they enter the acidic phago-lysosomal compartment of the cell.

Movie S7. Primary mouse microglia phagocytosis assay. Live imaging of primary mouse microglia (Hoechst-positive nuclei in grey) undergoing phagocytosis of human synaptoneurosomes tagged with pHrodo. Synaptoneurosomes can be seen in red as they enter the acidic phago-lysosomal compartment of the cell. 